\title{
Unexpected Emergent Behaviors From Elementary Swarms
}

\author{
Jon Roach, Winston Ewert and Robert J. Marks II \\ Baylor University \\ Dept. of Electrical \& Computer Engineering \\ Waco, Texas
}

\author{
Benjamin B. Thompson \\ The Pennsylvania State University \\ State College, PA
}

\begin{abstract}
Swarms are collections of loosely coupled distinct agents each following simple rules. Swarms do not use central coordination AND individual agents need not be aware of the swarm's overall function. As each agent performs its task, the swarm collective can display unusual and unexpected emergent behaviors. For those swarms defying analytic evaluation, simulation remains as the only method to reveal emergent behavior. A number of swarms are simulated each with no more than simple rules to follow. Each simulation reveals an interesting and often surprising emergent behavior. Termites clear areas and stack wood chips, gnats naturally confine themselves to swarm inside a circle of fixed area and sand piles develop instabilities and avalanche. Possibly the most interesting simulation is predator swarms pursuing swarms of prey in a game we dub bullies and dweebs. Individual bullies can be ineffective in killing dweebs, for example, whereas a mob of bullies can be highly effective. Addition of stochastic component to dweeb motion in a swarm is essential for prolonging dweeb life. The swarms are illustrated using screen shots of the swarm dynamics. More interesting and insightful videos of the swarming are available on NeoSwarm.com.
\end{abstract}

Index Terms-swarm intelligence, emergent behavior, computational intelligence, game theory

\section{INTRODUCTION}

Swarms [2] can be viewed from an engineering perspective as a collection of loosely coupled agents. Individually, the agents perform relatively simple tasks. The swarm's function is often characterized by an emergent behavior not apparent at the agent level. The functioning of the swarm is not simply a magnification of the agent's function, but can be unexpected. Legendary science fiction writer Michael Crichton centered his novel Prey [5] around an experientially adaptive man made swarm that went bad.

Termites are an example of a social insect swarm [11]. In one model the termites randomly move about on a surface covered by wood chips. When the termite bumps into a chip, it picks it up and continues its random meandering until it bumps into another wood chip whereupon it puts down the chip it is carrying. Now empty handed, the termite continues moving about until it bumps into another wood chip and the process is repeated. Individually, each termite is only aware of the immediate task at hand: walking around, bumping into a wood chip, and either picking it up if empty handed or dropping his load if carrying. At the level of the individual termite, the overall function of the swarm is not apparent.
The collective emergent behavior of the swarm is clearing the area and stacking wood chips. When the swarm function is identified, one can often stand back, look at the actions of the individual agents and see that stacking wood makes sense. We will be looking at simulations of this termite swarm later in the paper.

When teaching swarms to a class of students, a fun exercise is to have the students stand and secretly choose two of their colleagues in the class. For this swarm experiment, each student is asked to move in such a manner as to place themselves between the two students he has randomly chosen. As the student begins moving to do so, each of the students he has identified are themselves moving to place himself between the two students he has chosen. Before the experiment begins, the class is asked to identify the resulting emergent behavior of their actions. Typically most of the students have no idea. There is often one or more in the class, however, who can play the swarm in their heads and predict the outcome which is that all the students are attracted into a single group in the middle of the room. ${ }^{1}$

A related class experiment is to have the students again secretly choose two other students, $\mathrm{A}$ and $\mathrm{B}$, and move in such a manner that the student places B between himself and A. On first exposure, the emergent behavior of the entire swarm is not obvious. The result is that the students will all scatter and eventually be standing next to a wall in the room unable to move in such a way as to better their position. Although we have never done so, this would be an interesting exercise to perform outside where there are no walls. It would be an entertaining way to dismiss the class. ${ }^{2}$

Swarming is often characterized by simple performance rules imposed on each agent. In a somewhat similar vein, Stephen Wolfram in his highly promoted book A New Kind of Science [26] explores emergent behavior from simple

\footnotetext{
${ }^{1}$ There is the possibility that there will be two or groups in the case where the students choose in such a manner that groups are not connected Imagine, for example, separating the students into two rooms there they secretely choose two students who are in the same room with them. When the two groups are placed back into one room and the swarming action begins, there is no interaction between one group and the other. The steady state result will be the forming of at least two individual clusters.

${ }^{2}$ The examples using students were originally suggested by Bonabeau and Meyer [3] and analyzed by Gravagne and Marks [13]. Videos of simulations of these swarms are available on NeoSwarm.com.
} 
rules applied to cellular automata. Swarming and Wolfram's cellular automata both obey simple rules, yet can generate unexpected and complex emergent behavior. They also share the property that the emergent property is often not apparent from the rules.

Although swarms are typically associated with social insects, the idea applies as a paradigm to numerous other systems. Applications include economics [3], inverse problems [8], [14], [21], communications [1], [6], [7], [15], [18], optimization [9], [19], warfare [4], image processing [23], pattern recognition [27], [28], business [3], acoustics [25], national security [16], [17] and search algorithms [1], [20].

Swarms need not move. The lung, for example, can be modeled as a large number of disjunctive individually functioning cells each unaware of the actions of nonadjacent cells nor aware of the function or purpose of the collective. A fundamental emergent property of the lung is, of course, breathing.

Given the common disconnect between an agent's individual function and the swarm's emergent behavior, two approaches suggest themselves to develop deeper insight: analytic modeling and simulation. There are, indeed, interesting swarms whose behavior can be captured and described by mathematics [13]. Formulating simple agent behaviors that defy modeling is not difficult. Our aim is to explore the simulation analysis of the manner that swarms behave to hopefully sharpen through experience our ability to predict the emergent behavior from simple agent functions. In this print media, only screen captures of swarm dynamics can be presented. The reader is encouraged to view the parent videos to better appreciate and enjoy the swarm dynamics. They are available on NeoSwarm.com.

\section{TERMITES}

A simple model of a swarm of termites stacking wood was described in the INTRODUCTION. We consider two cases of termite swarms.

\section{A. Clearing \& Stacking}

Four snapshots of a termite swarm are shown in Figure 1. The initialization takes place at time $t_{0}$. Each small square denotes a wood chip. Each dot is an agent. Initially, all the agents are empty handed. The termites begin to move in random Brownian motion. If an agent hits an edge of the square playing field, it simply bounces off elastically.

At time $t_{1}$ the diamonds denote termites carrying wood. Clustering of wood chips into distinct piles is evident.

The wood is coded to be light blue initially. As time passes, the color changes to brighter yellow. Yellow squares, therefore, correspond to wood chips that have been there for a long time.

An alternate view of this swarm process is the clearing of wood chips. Consider the wood chip pile inside the box at time $t_{1}$. If the pile becomes destroyed, the creation of a future pile in the same area becomes improbable. An isthmus of sorts would need to stretch from another cluster into this region in order for this to occur. The boxed pile, it turns out, is ultimately dispersed to other piles. At time $t_{2}$ the termites have carried away all but one wood chip from the pile. At time $t_{3}$ this final wood chip has been removed and the region now is free of wood chips. It is doubtful that wood chips will ever again appear in this region.

\section{B. A Termite Swarm With Drift}

Another simulation of the termite algorithm is shown in Figure 2. There are two changes that have been made.

1) The termite playground is modular in the sense that termites exiting on the right appear at the same location on the left and continue their movement. This would be the case, for example, if the termites were operating around the cylindrical trunk of a tree. Likewise, termites leaving the top edge of the termite playground appear at the same horizontal coordinate at the bottom.

2) Termite motion, though still random, has a slight drift that nudges each termite towards the upper right of the playground.

As before, a termite and wood chip are not allowed to occupy the same space at the same time. This constraint has more importance here.

The initial stage of the swarm is shown at $t_{0}$ in Figure 2. The empty handed termite agents are shown as small white dots. Wood chips are shown in red and the cleared area in blue. Soon after initiation at time $t_{2}$, some of the termites are carrying wood chips. They are shown as white circles. In the beginning, the wood particles are scattered randomly and close together. The interval between picking up a wood chip and laying it down is short.

Due to the slight drift to the upper right, tunnels are beginning to form at time $t_{2}$. In the $t_{3}$ snap shot, boxes are drawn around two disabled termites. These disabled termites are carrying wood but are surrounded by wood. They neither have a place to put down their wood nor an open space to which to move. They therefore simply remain frozen where they are.

Two maverick termites are shown boxed at time $t_{4}$ in Figure 2. These termites are working their way through a solid wall of wood by picking up a wood chip before them and then placing it down behind. The effect is that the maverick termite slowly works its way through the wall of wood. As in the previous termite images, deposited wood changes color after it is first deposited and, in steady state, turns red. Monitoring the age of the wood in this manner allows us to see a trajectory behind the mavericks that indicate the path they have traversed. Maverick termites can reverse roles with the disabled. When a maverick bumps into a disabled termite, the disabled termite can steal the open space available to the maverick and begin to move again within the wood. The former maverick, robbed of its open space, becomes disabled.

The overall interesting emergent swarm behavior of the termites is evident at time $t_{4}$ in Figure 2 . As is the case in Figure 1, the termites are clustering wood. But because of the slight drift of the termites to the upper right, the clustering 

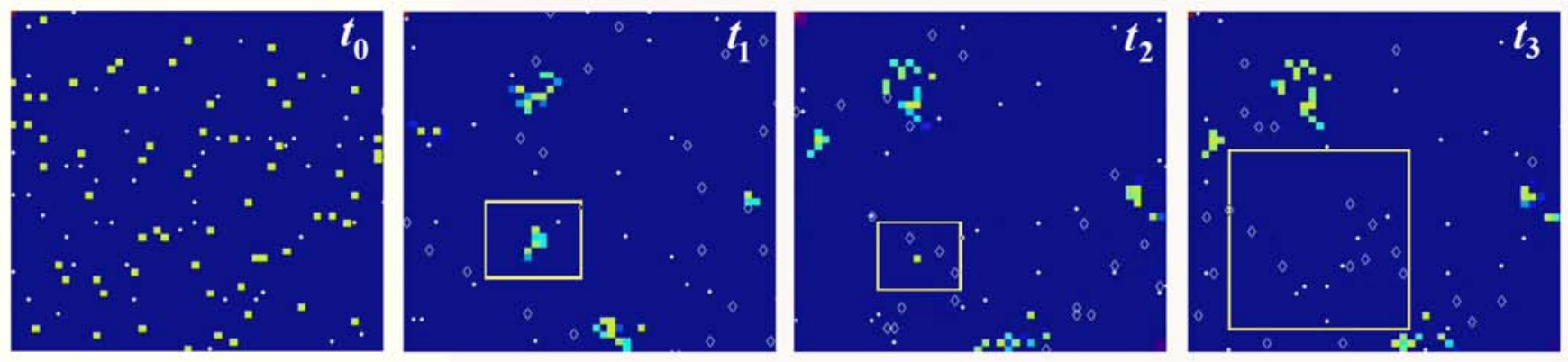

Fig. 1. Four snapshots of a termite swarm.
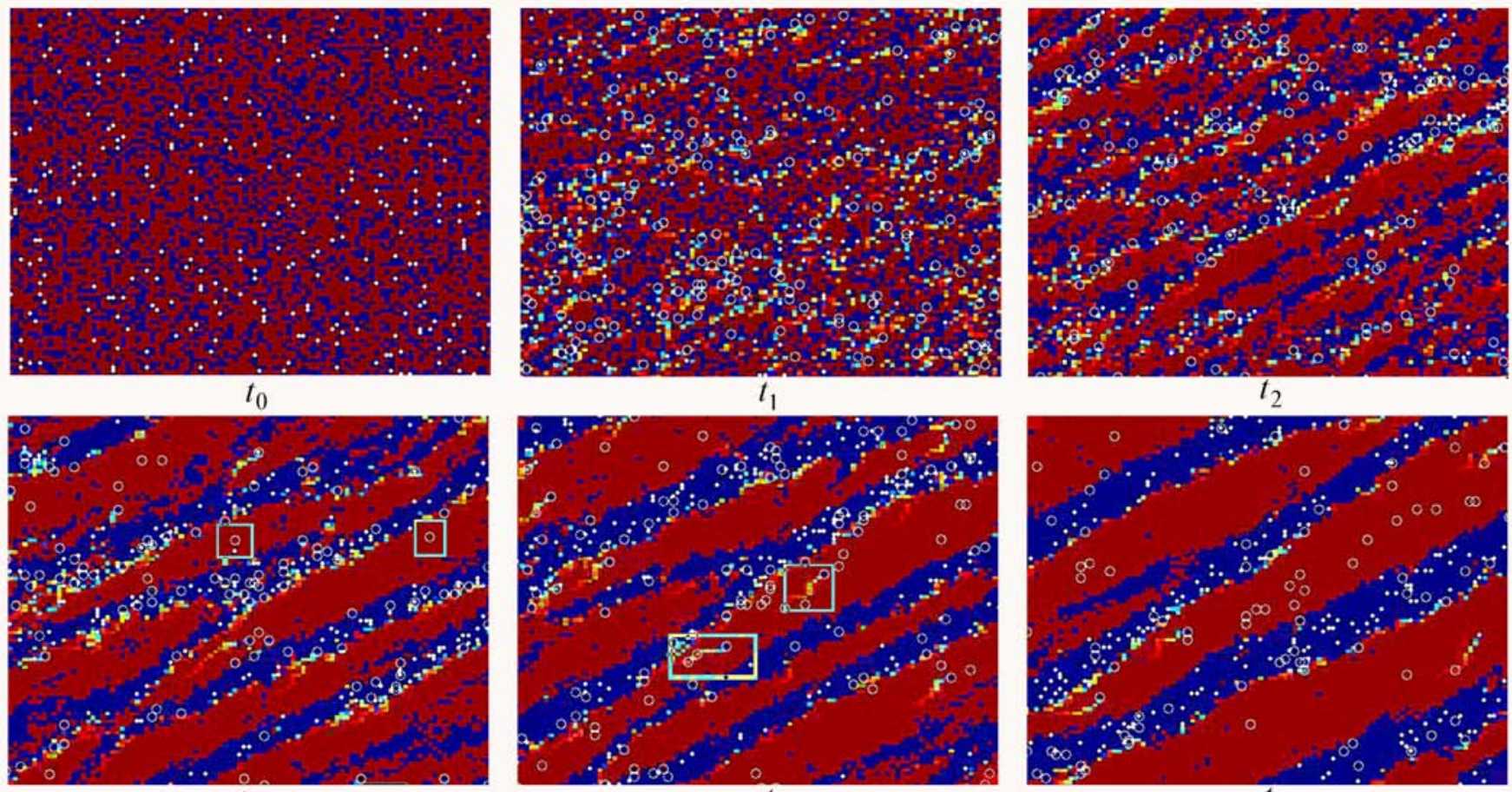

$t_{3}$
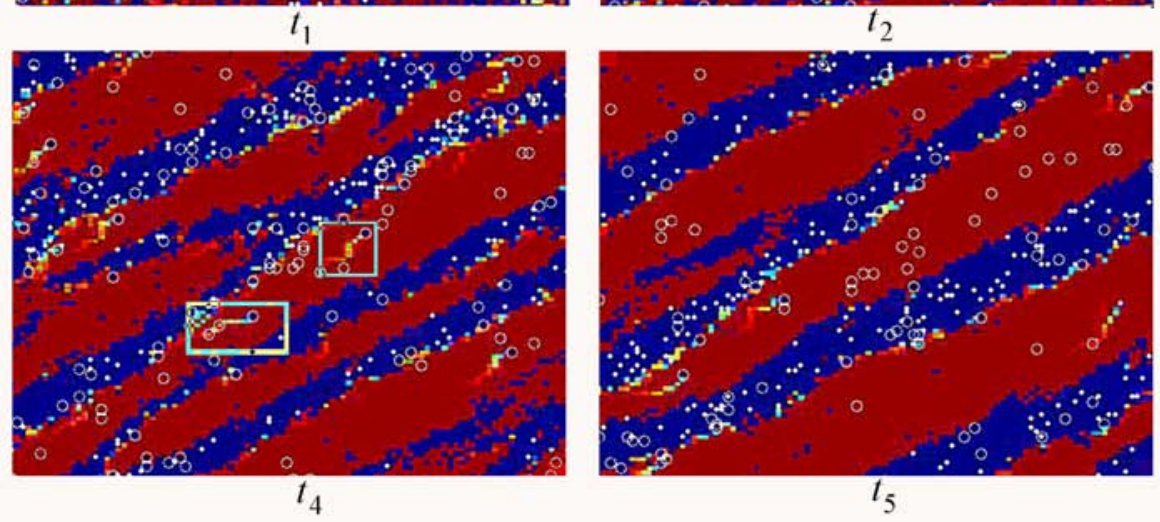

Fig. 2. Six snapshots of a termite swarm with drift.

is done in such a manner that roads are constructed. In the transition from $t_{2}$ to $t_{5}$, the roads are seen to become wider.

The interesting behavior of the termite simulations result only from two simple rules.

1) If a termite is empty handed and bumps into a wood chip, it picks it up and moves on.

2) If a termite is carrying a wood chip and bump into another wood chip, the termite drops the chip and moves on.

\section{GNATS}

Here are simple rules that simulate what looks to be a swarm of gnats. ${ }^{3}$ On a plane, choose a home point in the middle of the playground.

1) A number of gnats initially roam randomly (Brownian motion).

${ }^{3}$ No claim is made that this model relates to biology.
2) The gnat furthest from home point is given a drift component toward the home point.

The drift is the same as described in the termites example. If a gnat with drift is later again designated as the swarm agent furthest from the home point, its drift direction is reset.

These two simple rules give rise to a gnat like swarm shown in Figure 3(a) where the home point is shown by a small blue dot in the center of each image. The agent farthest from the home point on the right is circled in red.

A consequence of the rules is that the gnat swarm confines itself to a circle with finite radius. Typical of a gnat swarm is the snapshot shown in Figure 3(a). A plot of swarm radius versus time as measured by the gnat furthest from the home point is shown in Figure 4(a).

If the swarm agents are initialized in a more disperse manner as shown in Figure 3(b), the swarm will reduce in size to be commensurate to that in Figure 3(a). Likewise, if the 


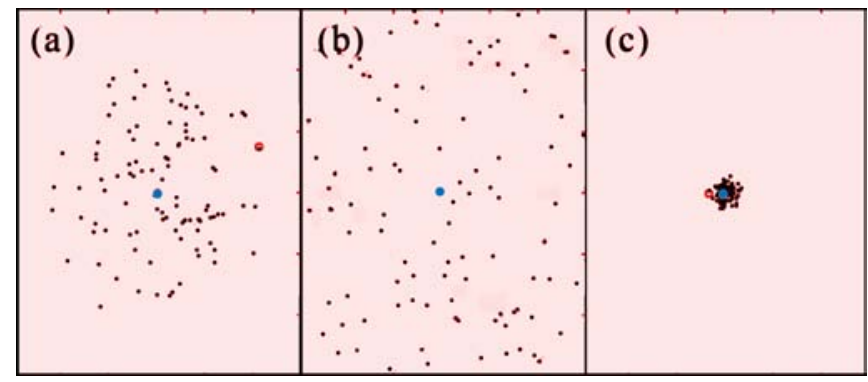

Fig. 3. Illustration of a swarm of gnats.

swarm agents are initially closely clustered as in Figure 3(c), the swarm radius will expand to a size similar to that in Figure 3(a). The expansion of the swarm radius in this case is plotted in Figure 4(b).

(a) Steady State Radius

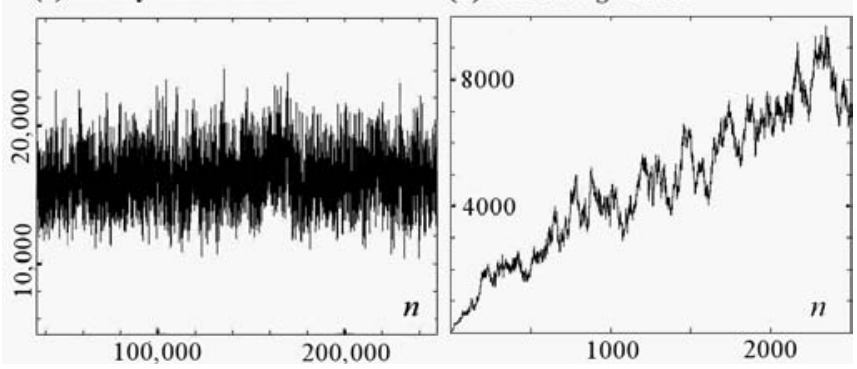

Fig. 4. Swarm radii for 200 gnats. Time, $n$, is not linear. Rather, each time a new gnat wins the contest of being furthest from the home point, its distance to the home point is recorded as the next point in the plot. These occurrences do not happen at equal intervals of time.

As is the case with other swarms following simple rules, unexpected behaviors emerge. In the case of the gnats, it is a confining circle of fixed radius.

\section{Avalanches On A Pile Of SAND}

In his book Ubiquity [4], Mark Buchanan explains application of the power law to earthquakes, market crashes, hurricanes, wars, personal income, city sizes and avalanches. Random variables obeying the power law are identified by plotting their probability density functions on log-log axes. If the plot is a straight line with negative slope, the underlying random variable is said to obey the power law [4], [24].

Some of the power law phenomena described by Buchanan fall within the swarm paradigm. One is piling sand. As sand is piled, small avalanches occur regularly. The rare event of a major avalanche is characteristic of phenomena obeying the power law.

The model we use to simulate a pile of sand follows simple rules.

1) Drop a grain of sand onto the middle of the sand pile.

2) Each grain of sand has an elevation above the sandbox floor. The new grain looks at its adjacent neighbors. If there is a lower position available, the grain moves to that position. (If there are two or more lower locations, one is chosen at random.) The new location of the
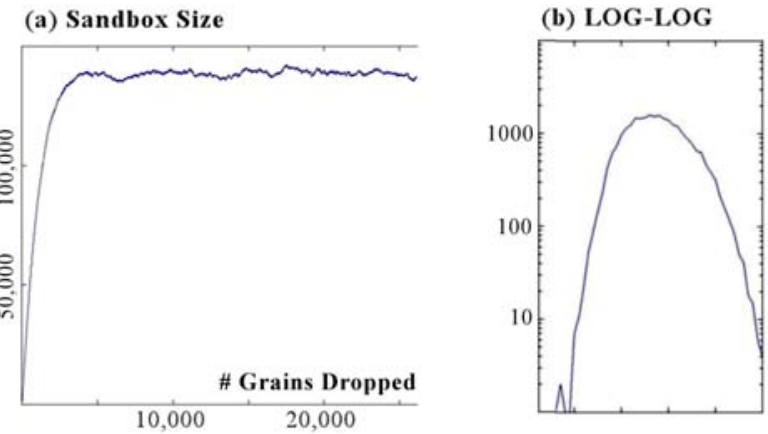

Fig. 6. Sand pile data.

grain might, itself, be higher in elevation than its surroundings, in which case the grain of sand is again moved to the lower position. This process is repeated until no more sand moves.

3) Go to the first step and repeat.

The total number of displacements that result from the dropping of a single grain of sand is a measure of the strength of the avalanche. In most cases, the number is small. There is a chance, however, the history of dropped sand has built an instability in the pile that will be unleashed as a major avalanche when the next grain of sand is dropped.

The pile of sand can be viewed as falling within the swarm paradigm. There are individual agents following simple rules. In addition each grain interacts only with its immediate neighbors and the grains of sand have no oversight awareness of the behavior of the larger system in which they function.

Screen shots of the simulation of the sand pile following these sand pile rules are shown in Figure 5. The two images on the left show the initiation and initial growth of the sand pile. The image on the right is characteristic of the sand pile in steady state. Any grain of sand near the edge of the table can be nudged off of the table and be lost to the system. For this reason the number of grains of sand in the pile in steady state is approximately constant. This is shown in Figure 6(a) where, after ramping up, the plot of the total number of grains of sand in the sandbox flattens.

A log-log histogram of avalanche size is shown in Figure 6(b). The number of grains of sand in an avalanche does not decrease as the frequency of occurrence increases. Small avalanches of one or two displacements are rare. Indeed, there is a maximum likelihood avalanche size located at the maximum of the histogram curve. To the right of this maximum, however, the downward almost linear behavior of the histogram suggests that, in this region at least, the idea of the power law may be in effect.

\section{Bullies \& DWEeBS}

Bullies and dweebs is a simple predator-prey swarm with simple rules. The emergent behaviors in this game are the most fascinating and richly diverse of the examples discussed thus far.

Here are the rules. On a battleground, each predator bully will identify the closest dweeb and take a step in the dweeb's 


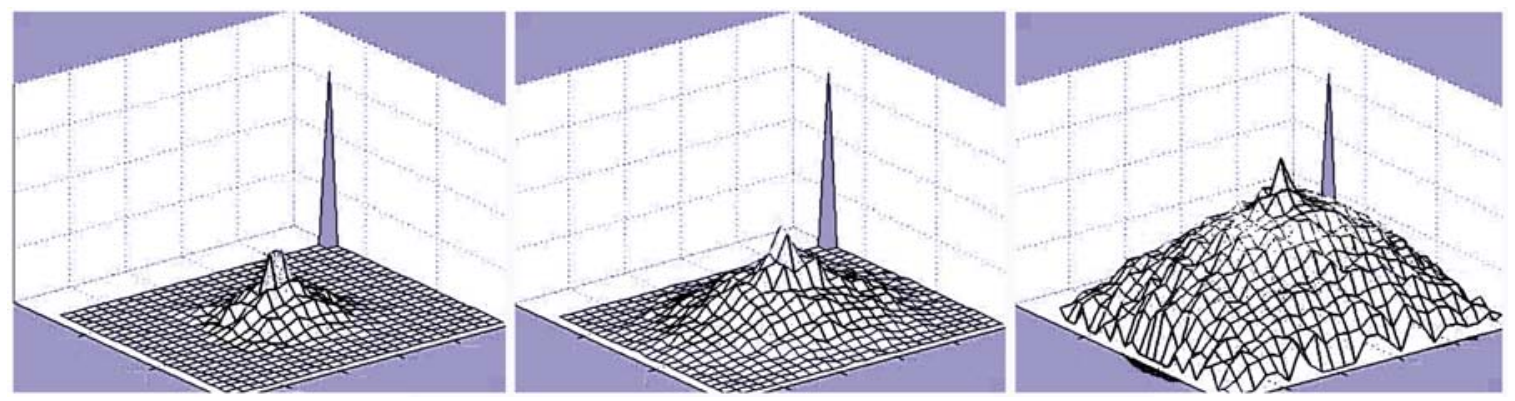

Fig. 5. Forming a sand pile. (The spike in the back corner is an artifact of the simulation software that keeps the scaling of the pile the same from frame to frame. It has otherwise nothing to do with the problem.)

direction. Likewise, each dweeb detects the closest bully and takes a step away from the bully. When a bully catches a dweeb, the dweeb is removed from the game and the killing bully immediately seeks the next closest dweeb. The walls of the battleground are hard. Neither bully nor dweeb can pass through walls.

Using these simple rules results in rich variations of emergent properties.

\section{A. Entrapment}

Snapshots from a fundamental game of bullies and dweebs are shown in Figure 7. Initialization time $t_{0}$ begins with a large number of randomly positions dweebs indicated by the blue squares. Bullies are red diamonds.

At time $t_{1}$ there are two lines of dweebs moving upwards pursued by bullies on the bottom. The bullies are unaware there is a line of dweebs before them. They are only interested in the pursuance of the nearest dweeb. The dweebs in a line running from the bully, on the other hand, are are aware of the bully. The bully is the nearest to them and their job is to run away. Two swarms of dweebs to the right of these two bullies are likewise running away from the bullies.

Apparent cooperation between two bullies occurs at time $t_{2}$. As shown by the red arrows, one bully is chasing a line of dweebs downwards while another is chasing a line of dweebs upwards. The dweebs are trapped between the two bullies and are eventually killed. Although the bullies appear to be cooperating, neither bully is directly aware the other bully is there. They are only aware of the nearest dweeb who they are pursuing.

The swarm at time $t_{3}$ in Figure 7 illustrates a common property of predictor-prey games. Two bullies are moving in the direction of the red arrows in the pursuance of the dweebs. The dweebs will eventually be trapped on the walls where they will be killed by the bullies.

1) Alexander the Great's Defense Against Swarms: Entrapment, an emergent behavior in the bullies and dweebs game, is as an effective countermeasure to swarm attacks in warfare. In his book about swarms on the battlefield, author Sean Edwards writes [10]

"Alexander the Great was one of the first Western military commanders to encounter an enemy who used swarming tactics. The Scythians, a nomadic people who generally fought with horse archers and used swarming tactics, turned out to be the first army to defeat the Macedonian phalanx after it crossed the Hellespont. However, Alexander improvised new tactics to counter the swarming tactics of the Scythian horse archers and eventually defeated them."

As the bullies trapped the dweebs against the wall, so Alexander trapped the Scythians. Edwards writes

"Alexander realized that the best way to come to grips with the more mobile Scythians was to pin the swarmer against an obstacle, such as a river or a fort. Since a geographic obstacle was not at hand, Alexander used his own men as bait by sending a cavalry force forward before his main army to provoke the hostile horse archers into attacking ... Once the Scythians had swarmed and circled around Alexanders cavalry bait as expected, Alexander brought forward his light infantry to screen the advance of his main cavalry force. Fuller logically assumes that the subsequent cavalry charge was aimed at the Scythians trapped between the light infantry and the bait force. Over 1,000 Scythians were killed and 150 captured in this battle, although the main part of the horse archer army escaped. The Scythians sued for peace shortly thereafter."

Note there were massive killings after entrapment. We will see this occurs repeatedly as the bullies pin dweebs against walls or in corners.

2) Limitations: The deterministic nature of the bullies and dweebs in this example has limiting attributes.

- A swarming appearance is discouraged. Bullies, for example, chase dweebs that are constrained to be in a line.

- Bullies stack. If two bullies coincidentally land on top of each other, they will stay that way for the remainder of the contest. Both bullies see the same nearest dweeb and will both move towards it in exactly the same way.

- Dweebs stack. Similarly, if two dweebs occupy the same coordinate, they will stay that way for the remainder of the game. Both see the nearest bully and both react identically in their defensive motion.

Bully stacking inhibits the attacking capability of the bully collective. Dweeb stacking limits the overall ability of dweebs to evade a bully. And when there is a kill of a stacked dweeb, a large number of dweebs die at the same time. 

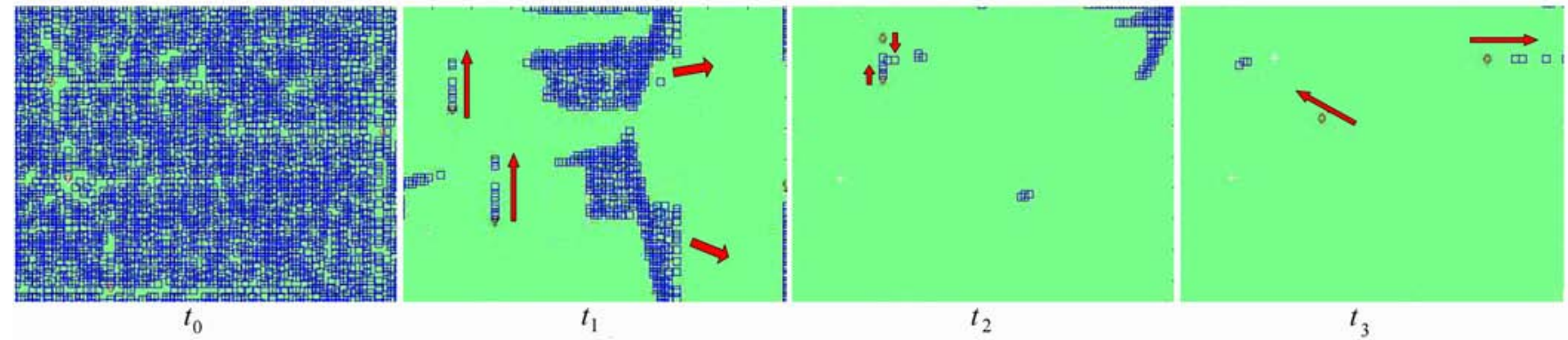

Fig. 7. Snapshots of a fundamental bullies \& dweeb game. The blue squares are dweebs and the red diamonds are bullies. Initially there are 5000 dweebs and three bullies.

\section{B. Adding Dweeb Twiddle}

The swarm limitations observed in the previous section can be addressed by adding a small random component to the movement of the agents. We call this random component twiddle. First, we will add twiddle to the dweebs. As before, the dweeb moves away from the bully but now a small random direction added to each retreating step. Otherwise the rules remain as before. (Since twiddle has not yet been applied to the bullies, bully stacking can still occur.) As is illustrated in Figure 8, simple addition of twiddle changes the swarm dramatically. The dweeb swarm ${ }^{4}$ moves more as a cloud rather than a structured group like dweebs-in-a-row. Twiddle allows the dweebs to more effectively avoid the bullies by forcing the bullies to concentrate on a single dweeb while the other dweebs scatter.

Some of the dynamic changes resulting from dweeb twiddle are illustrated in Figure 8. At time $t_{1}$, Bully B is chasing a swarm of dweebs down the battleground. The twiddle allows the dweebs to swarm and escape the strict straight line structure characteristic when the dweebs had no twiddle.

At time $t_{1}$, Bully $\mathrm{A}$ is about to hit the right wall. Like trapped Scythians, a number of dweebs are lined up against the right wall to get as far away from the bully as possible. There is a slight downward drift of the walled dweebs as Bully A approaches from the left. When Bully A hits the right wall, two interesting thing happen.

- First, only a single dweeb is killed. This is the single dweeb on which Bully A has had his eye. ${ }^{5}$ Dweeb twiddle separates the dweebs from each other and makes this possible.

- Second, all of the dweeb wall flowers on the right flee from the wall. This is shown at time $t_{2}$. Twiddle allows them to separate from the wall and realize there is an option other than staying glued to the wall. Without twiddle, a walled dweeb can only step away from a walled bully by moving up or down the wall. Twiddle allows a small step away from the wall and allows the dweeb to run in the open field. The dweeb swarm is running from Bully A. While Bully A pursues the

\footnotetext{
${ }^{4}$ Cattle comes in herds, fish in schools and beer in six packs. A collection of dweebs will be henceforth be referred to as a swarm. A group of bullies will be called a $m o b$.

${ }^{5}$ Yes. All bullies are male.
}

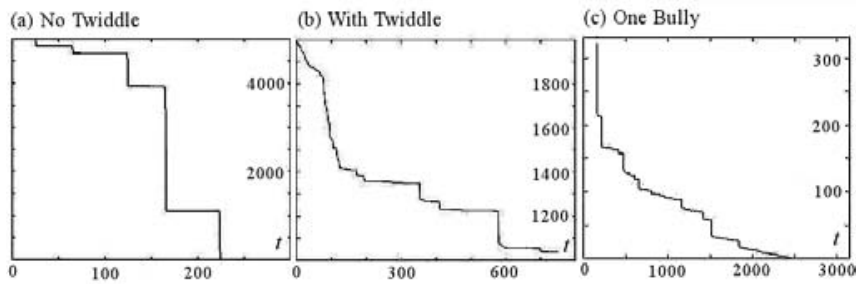

Fig. 9. A plot of the number of surviving dweebs as a function of time for three different scenarios.

closest dweeb and moves down the right wall, the other dweebs scatter from Bully A in a swarming fashion. This retreating direction will continue until dweebs detect Bully B as being the closest whereupon a different retreat strategy will be adopted.

The role of twiddle in allowing dweeb survival is also illustrated at time $t_{3}$ in Figure 8. Bully $\mathrm{A}$ is chasing what appears to be a single dweeb into the lower right corner. The apparent single dweeb is, in fact, a large number of dweebs that, despite twiddle, are spaced closely together. As shown at time $t_{4}$, when Bully $\mathrm{A}$ hits near to the lower right corner, all of the dweebs in the boxed region explode away from the corner to run from Bully A.

The location on the battleground where dweebs were previously killed are marked by small subtle white +'s in Figures 7- 8. In Figure 7 where no dweeb twiddle is used, there are few mid field kills. The few shown happened primarily at the initialization of the game. Without twiddle, most of the kills occur at the boundaries. This can be seen in Figure 9(a) where a plot of surviving dweebs as a function of time is shown. The plot has numerous rapid drops each corresponding to the death of many dweebs at one time. All occur at the boundaries of the battlefield. Dweeb stacking is responsible for these drops since a kill of a single stack corresponds to the death of all of the dweebs in the stack. When dweeb twiddle is added, stacking only occurs by collocation coincidence. As seen in Figure 8, there are many more mid field kills although most of the kills still occur at the battleground boundaries. The surviving dweeb versus time plot is shown in Figure 9(b). Because only one dweeb is killed at a time, the curve is smoother. Some fast drops are still present corresponding to mass killings at the battleground 

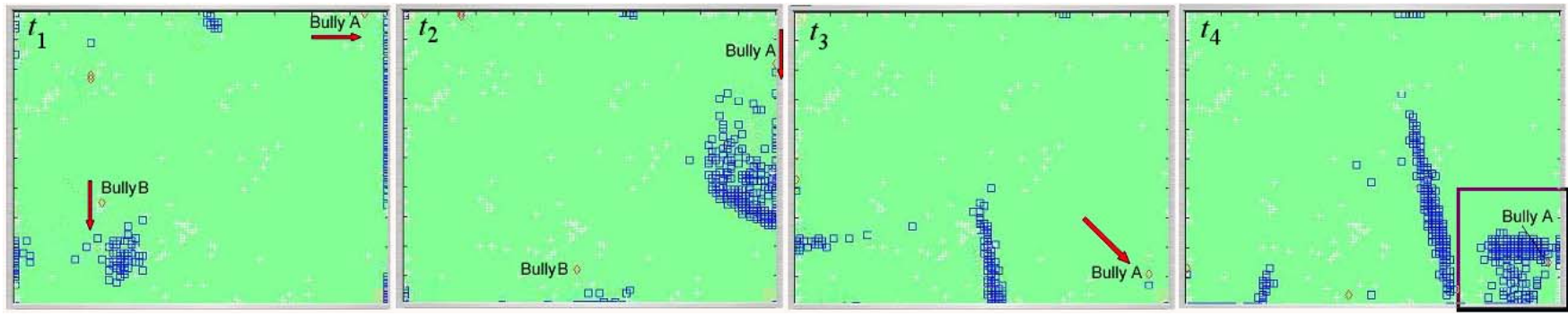

Fig. 8. Snapshots of a fundamental bullies \& dweeb game where the dweebs have twiddle. The initial distribution of bullies and dweebs was similar to that shown at time $t_{0}$ in Figure 7. The swarm starts with 2000 dweebs and 25 bullies. During the warming, some of the bullies stack.

boundaries.

1) One Bully : Figure 10 uses the same scenario except we use only one bully. ${ }^{6}$ Kills typically occur at the walls of the battleground. After each kill, the bully detects the nearest dweeb, chases the dweeb to another wall where the next kill occurs. There is typically one kill each time the bully crosses the battleground and hits another wall. The dweeb twiddle impedes greater slaughter by dispersing the dweebs into a swarm.

At time $t_{1}$ in Figure 10, the bully is close to a kill in the upper left of the top boundary. After the kill, the bully chases another dweeb to the right to the next kill that occurs on the top of the right hand boundary. Times $t_{3}$ and $t_{4}$ show the bully's pursuit of the next dweeb towards the bottom boundary of the battleground. Notice the dweeb swarming in these two pictures. At $t_{3}$, the dweebs are beginning to run from the right hand wall to escape the bully. At time $t_{4}$, they are all on the bottom boundary as far south as they can get from the bully.

Although most kills occur at the boundaries of the battleground, there are exceptions. One is shown at time $t_{5}$ in Figure 10 where the bully is shown to be directly on top of a dweeb. After the kill, the bully proceeds to the left wall where another dweeb is killed. Then as shown at time $t_{6}$, the bully then turns around and pursues the next dweeb for a kill on the right hand wall. In the mean time at times $t_{5}$ and $t_{6}$, the dweebs not being pursued continually position themselves to be as far away from the bully as possible.

As is seen by comparing Figure 9(b) with Figure 9(c), one bully takes a significantly longer time to totally eliminate the dweebs. And because there is typically only one killing for each time the bully crosses the battleground, the plot of the surviving dweebs reduces almost linearly after about time $t=200$. Before $t=200$ there were still transients in effect.

2) Paralyzed Bullies and Voronoi Partitioning : When the bullies are frozen in position, dweebs will position themselves on the Voronoi partition boundary [12], [22]. These boundaries are a function of the positioning of the bullies. The Voronoi partition is illustrated in Figure 11. On the left are eleven designated points on a plane. Every other point on the plane is assigned to the designated point to which

\footnotetext{
${ }^{6}$ Actually, there are two bullies who soon became stacked into the equivalent of one bully. All of the snapshots in Figure 10 are for the stacked bully.
}
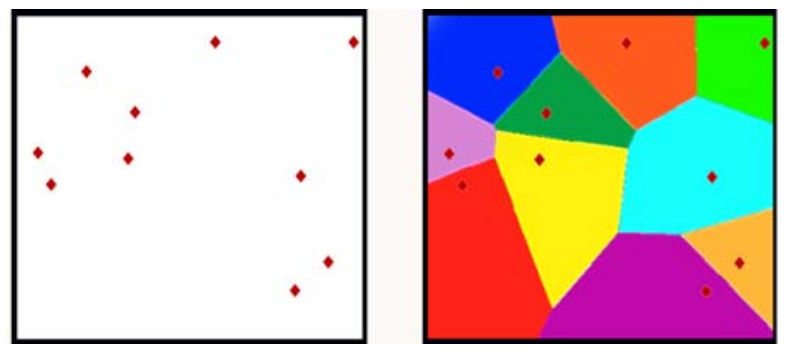

Fig. 11. A positioning of bullies is shown on the left. On the right is the Voronoi partition of these points.
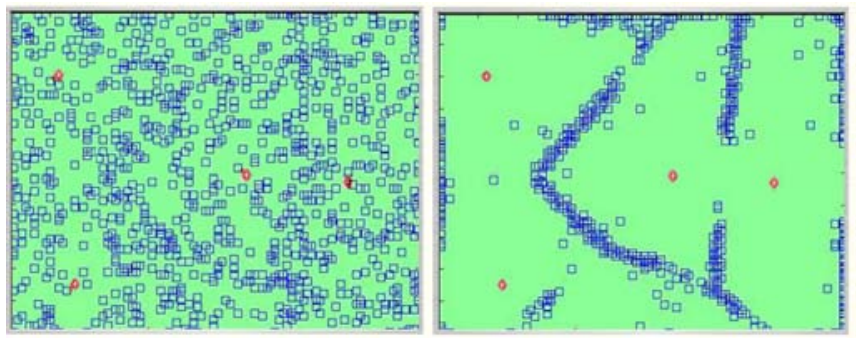

Fig. 12. Voronoi partitioning performed in the bullies and dweebs swarm.

it is nearest. This results in the Voronoi partition shown on the right. ${ }^{7}$

As illustrated by the screen shots in Figure 12, Voronoi partitioning occurs in the bullies and dweebs game when the bullies are frozen in position. On the left is the swarm initialization and on the right is the Voronoi partition that occurs soon after the swarm is started. In hindsight, this behavior makes sense. Each dweeb positions itself to retreat from the nearest bully. At the Voronoi boundary, the dweeb is equally distant from the two bullies who define the boundary.

\section{Bullies With Twiddle}

We continue with the bullies and dweebs example using the same two basic rules: Each dweeb steps away from the nearest bully. Each bully steps towards the nearest dweeb. (The variations and richness of the emergent behaviors behooves us to occasionally remind ourselves of the simplicity of the rules that define the contest.) Adding twiddle to the

\footnotetext{
${ }^{7}$ This figure is derived from a public domain image from Wikimedia Commons.
} 


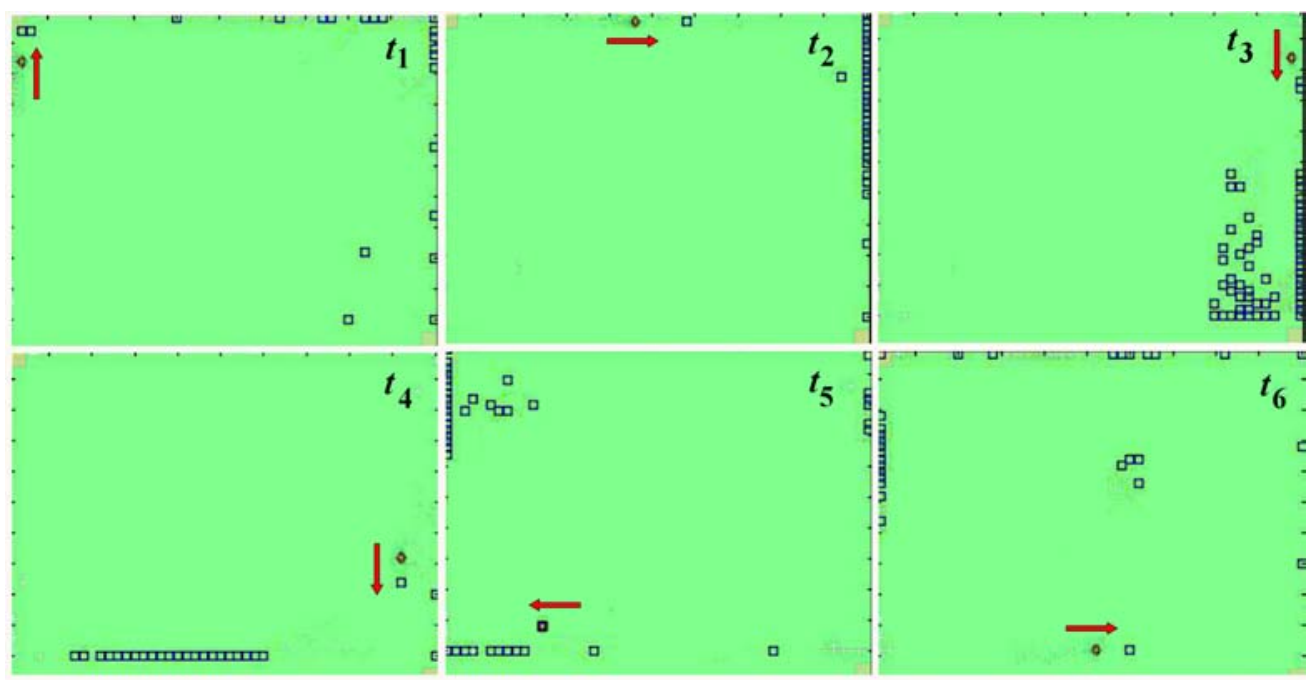

Fig. 10. Snapshots of the actions of one bully on dweebs with twiddle. Usually, but not often, kills are at a wall.

dweeb motion allows the dweebs to survive longer. What happens when the we add twiddle to the bullies?

Dependent on the number of bullies in the pursuit, using bully twiddle can either be highly detrimental to the bully's effectiveness or highly beneficial. It depends on the number of bullies participating in the pursuit. Isolated bullies with twiddle appear disoriented. Their drunken swagger still allows them to get close to a dweeb, but inhibits their ability to make the final kill. A large collection of drunk bullies on the other hand becomes a destructive mob who collectively are highly effective in killing dweebs.

1) When Twiddle Makes Bullies Tipsy : In the video available at NeoSwarm.com, the actions of the isolated tipsy bully is almost comical. The behavior illustrated in the screen captures in Figure 13, although instructive, does not capture the fun. The snapshots in Figure 13 are looking at section next to the lower right wall of the battleground where a bully with twiddle pursues a single dweeb with twiddle. Times $t_{1}$ through $t_{3}$ show the bully pushing the dweeb towards the right wall. Normally this would be sufficient for a kill. Frames $t_{4}$ through $t_{6}$ show the dweeb escaping. The twiddle in the bully makes it too unsteady to deal the death blow. At times $t_{7}$ through $t_{9}$, the pursuance of the bully and avoidance of the dweeb has broken away from the wall into open field which is advantageous to the dweeb. The randomness of the twiddle of both agents, however, moves the pursuit back to the right hand wall of the battleground at times $t_{10}$ and $t_{11}$. The inebriated bully, it turns out, is still unable to make the kill and the dweeb breaks away from the wall to a less dangerous open field pursuit.

2) Drunken Mobs of Bullies : When there are a large number of bullies with twiddle, the collective can act together as an effective dweeb killing mob. An illustration is shown in the screen shots in Figure 14. Initially the bullies are randomly placed and, acting individually, are ineffective in their killing mission. As the game plays, the bullies self organize into mob clusters. Since the dweebs are already traveling in swarms, the bullies each tracking the same or different dweebs within the dweeb swarm themselves form a cluster which, in the case of bullies, we will call a mob. Bully mobs are evident at time $t_{1}$ in Figure 14. Red arrows adjacent to bully mobs indicate the general direction in which the mob is moving. At time $t_{2}$ the two mobs labeled with arrows are pursuing different dweeb targets. At times $t_{3}$ and $t_{4}$, the bully mob labeled by the leftmost arrow is chasing a dweeb into the left wall. Once this dweeb is killed, the bully mob's attention is refocused on another dweeb cluster as seen at time $t_{5}$. At times $t_{6}$ and $t_{7}$, all bully mobs begin to move towards the upper left corner where the target dweeb swarm is trapped. Once the dweeb swarm is killed, the bully mob moves to its new dweeb swarm target at time $t_{8}$. Note that the disjoint bully mobs at the beginning at time $t_{1}$ have now joined into a single large mob. Generally, bully mobs both conglomerate and bifurcate during their pursuit of the dweebs.

3) A Dweeb Massacre: Another illustration of the power of the bully mob is illustrated in Figure 15. The contest begins at time $t_{0}$ with all of the bullies located in the upper left hand corner. The dweebs are likewise all clumped in the same location. At time $t_{1}$, the bully mob begins pursuit of the dweeb swarm towards the lower right corner. Due to twiddle, both the bully mob and the dweeb swarm begin to spread. At time $t_{2}$, the fate of the dweebs becomes evident. They are doomed to be trapped and massacred in the lower right corner of the battleground as shown at time $t_{3}$. But something interesting now happens at time $t_{4}$. A lone dweeb escapes the massacre and begins to run from the bully mob. The bully mob begins to pursue as is seen at time $t_{5}$. Ultimately, the bully mob catches the lone dweeb at the top edge of the battleground.

\section{Drive fast. Turn left}

NASCAR drivers, like swarm agents, are said to follow two simple rules: 


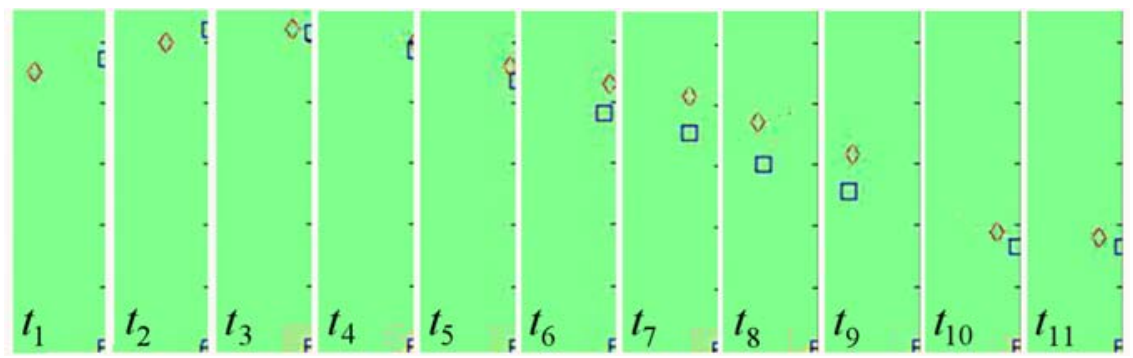

Fig. 13. Behavior of a bully and dweeb along the right wall of the battleground. Two dweebs in the lower right corner stay where they are at and are seen in each snapshot.

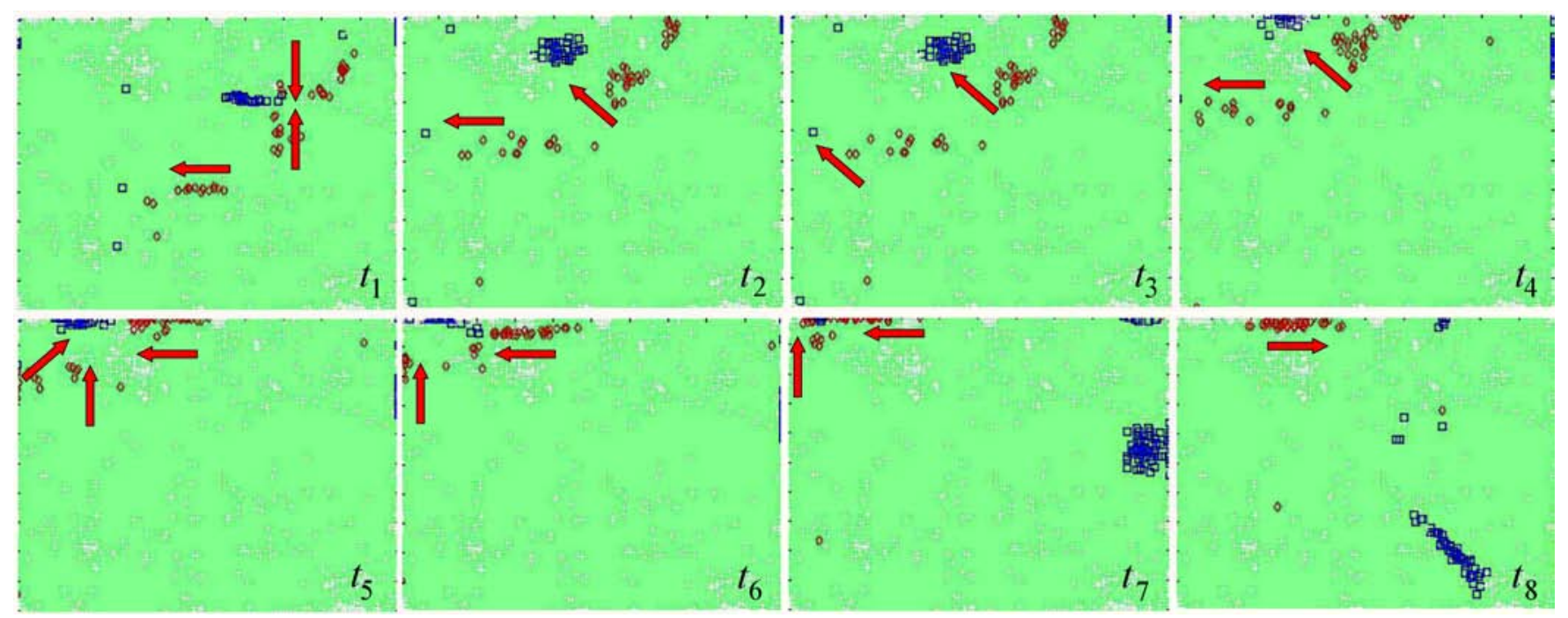

Fig. 14. Mobs of bullies pursuing swarms of dweebs.
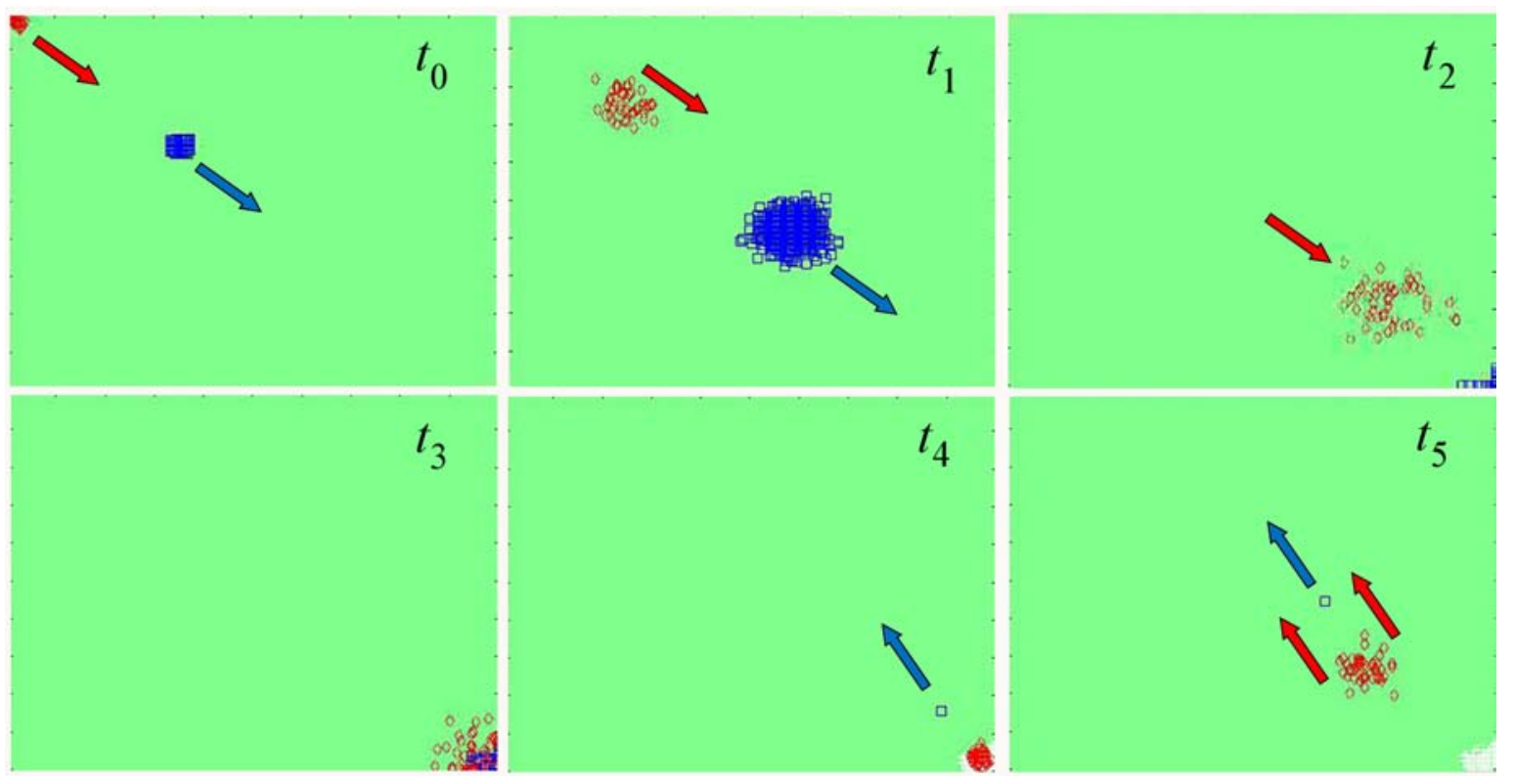

Fig. 15. A massacre of dweebs with a lone survivor. 
1) Drive fast.

2) Turn left.

All of the emergent behaviors illustrated in Figures 7 through 15 result from two rules: each bully steps toward the nearest dweeb and each dweeb steps away from the nearest bully. None of the agents is aware of the behavior of the swarm as a whole. They are aware only of their own individual actions. The wide variation in emergent behaviors is due to

1) the presence or absence of twiddle,

2) the number of participating agents,

3) the boundary conditions of the battleground, and

4) the initial placement of the agents.

\section{Conclusions}

Swarms obeying simple rules can display interesting and unexpected emergent behaviors.

Involvement with swarm simulations can be mildly addictive. We have found ourselves rooting for a lone brave dweeb running from a bully mob like we would root for a favorite sports team. The lone drunken bully trying unsuccessfully to capture a wile dweeb is truly amusing. This fascination with swarms springs from the unknowable and often surprising emergent behaviors.

When presenting these results in a lecture, there are invariably questions of the form "Did you try such and such?" or comments such as "It looks like you could have done such and such this way to achieve better or more interesting results." Viewing swarm activity invariably invokes ideas for variations. For those so inclined, feel free to tweak the MATLAB code used to generate these swarms. Most are available at NeoSwarm.com.

All of the examples given in this paper are from simulation analysis. The more difficult design or inverse problem is to generate desired emergent behavior by choosing appropriate swarm rules. We are looking into this problem. The interested reader is referred to our recent paper for some preliminary results [11].

\section{REFERENCES}

[1] P. Arabshahi, Andrew Gray, I. Kassabalidis, Arindam Das, Sreeram Narayanan, M. El-Sharkawi \& R.J. Marks II, "Adaptive Routing in Wireless Communication Networks Using Swarm Intelligence," Proc. 19th AIAA Int. Communications Satellite Systems Conf., 17-20 April 2001, Toulouse, France.

[2] E. Bonabeau, M. Dorigo, and G. Thereulaz, Swarm Intelligence: From Natural to Artificial Systems. New York: Oxford Univ. Press, 1999.

[3] E. Bonabeau and C. Meyer, "Swarm intelligence, a whole new way to think about business," Harv. Bus. Rev., vol. 79, no. 5, pp. 106114, May 2001

[4] Mark Buchanan, Ubiquity: The Science of History $\cdots$ or Why the World Is Simpler Than We Think, Crown ( 2001)

[5] Michael Crichton, Prey. Avon (2003)

[6] A.K. Das, R.J. Marks II, M.A. El-Sharkawi, P. Arabshahi, and A. Gray, "The minimum power broadcast problem in wireless networks: an ant colony system approach," Proc. IEEE CAS Workshop on Wireless Communications and Networking, Pasadena, CA, Sept. 5-6, 2002.

[7] A.K. Das, R.J. Marks II, M.A. El-Sharkawi, Payman Arabshahi and Andrew Gray, "r -shrink: A Heuristic for Improving Minimum Power Broadcast Trees in Wireless Networks," IEEE Global Telecommunications Conference 2003. GLOBECOM '03. ,Volume: 1, 1-5 Dec. 2003, pp.523 - 527.
[8] Russell W. Duren, Robert J. Marks II, Paul D. Reynolds and Matthew L. Trumbo, "Real-Time Neural Network Inversion on the SRC-6e Reconfigurable Computer," IEEE Transactions on Neural Networks, vol. 18, no. 3, May 2007 pp. 889-901.

[9] R. C. Eberhart and Y. Shi, "Comparing inertia weights and constriction factors in particle swarm optimization," in Proc. Congr. Evol. Comput., 2000, vol. 1, pp. 8488 .

[10] S. J. A. Edwards, Swarming on the Battlefield Past, Present, and Future. Santa Monica, CA: RAND Corp., 2000.

[11] Winston Ewert, Robert J. Marks, II, Benjamin B. Thompson and Albert Yu. "Evolutionary Inversion of Swarm Emergence Using Disjunctive Combs Control," IEEE Transactions on Systems Man \& Cybernetics: Systems (2013).

[12] Marina L. Gavrilova, Editor, Generalized Voronoi Diagram: A Geometry-Based Approach to Computational Intelligence. Springer 2008.

[13] I. A. Gravagne and R. J. Marks, II, "Emergent behaviors of protector, refugee and aggressor swarm,'IEEE Trans. Syst., Man, Cybern. B, Cybern., vol. 37, no. 2, pp. 471476, Apr. 2007.

[14] C.A. Jensen, R.D.Reed, R.J.Marks II, M.A. El-Sharkawi, Jae-Byung Jung; R.T. Miyamoto, G.M. Anderson, C.J. Eggen, "Inversion of feedforward neural networks: algorithms and applications," Proceedings of the IEEE, Volume: 87 9, Sept. 1999 , pp. $1536-1549$

[15] I. Kassabalidis, M.A.El-Sharkawi, R.J.Marks II, P. Arabshahi, A.A.Gray, "Swarm Intelligence for Routing in Communication Networks," IEEE Globecom 2001, Nov. 25-29, 2001, San Antonio , Texas.

[16] Ioannis N Kassabalidis, Mohamed El-Sharkawi, Robert J. Marks II, "Dynamic Security Border Identification Using Enhanced Particle Swarm," IEEE Transactions on Power Systems, Volume: 17 Issue: 3, Aug. 2002, pp. $723-729$.

[17] I.N. Kassabalidis, Mohamed El-Sharkawi, Robert J. Marks II, "Border Identification For Power System Security Assessment Using Neural Network Inversion: An Overview," 2002 Congress on Evolutionary Computation, 2002 IEEE World Congress on Computational Intelligence May12-17, 2002, Honolulu, pp.1075-1079.

[18] I. Kassabalidis, M. El-Sharkawi, R. J. Marks, II, P. Arabshahi, and A. Gray, Adaptive-SDR: Adaptive swarm-based distributed routing, in Proc. Int. Joint Conf. Neural Netw., Honolulu, HI, May 1217, 2002, pp. 351354.

[19] J. Kennedy and R. C. Eberhart, Swarm Intelligence, Morgan Kaufmann, 2001

[20] Russell D. Reed and Robert J. Marks II, "An Evolutionary Algorithm for Function Inversion and Boundary Marking," Proceedings of the IEEE International Conference on Evolutionary Computation, p. 794797, November 26-30, 1995

[21] Paul D. Reynolds, Russell W. Duren, Matthew L. Trumbo \& Robert J. Marks II, "FPGA Implementation of Particle Swarm Optimization for Inversion of Large Neural Networks," Proceedings 2005 IEEE Swarm Intelligence Symposium. SIS 2005. June 8-10, Pasadena, pp. 389 392.

[22] Steven S. Skiena, The Algorithm Design Manual 2nd edition, Springer 2008. p.576

[23] David Sturgill, Benjamin Van Ruitenbeek hatand Robert J. Marks II, "Image Compression and Recovery through Compressive Sampling and Particle Swarm," Proceedings of the 2009 IEEE International Conference on Systems, Man, and Cybernetics. San Antonio, TX, USA - October 2009, pp.1822-1826.

[24] M.P.H. Stumpf and M.A. Porter, "Critical Truths about Power Laws," Science vol. 335, 2012, pp.665-6.

[25] Benjamin B. Thompson, Robert J. Marks II, Mohamed A. El-Sharkawi, Warren J. Fox, and Robert T. Miyamoto, "Inversion of Neural Network Underwater Acoustic Model for Estimation of Bottom Parameters Using Modified Particle Swarm Optimizers," 2003 International Joint Conference on Neural Networks, July 20-24, 2003 , Portland, Oregon (pp. 1301-1306).

[26] Stephen Wolfram, A New Kind of Science. Wolfram Media, Inc. 2002. Available online at http://www.wolframscience.com/.

[27] R. Xu, J. Xu, and D.C. Wunsch II, "Clustering with differential evolution particle swarm optimization," in Proc. World Congress on Computational Intelligence (WCCI) 2010, Barcelona, Spain, 2010.

[28] R. Xu, J. Xu, and D.C. Wunsch, "A Comparison Study of Validity Indices on Swarm Intelligence-Based Clustering," IEEE Trans. on Systems, Man and Cybernetics, part B, Vol. 42, No. 4, pp. 1243 1256, 2012. 